\title{
Acute glucose fluctuation promotes in vitro intestinal epithelial cell apoptosis and inflammation via the NOX4/ROS/JAK/STAT3 signaling pathway
}

\author{
BINGYU CHEN ${ }^{1,2^{*}}$, YUANYUAN JIA ${ }^{3 *}$, DONGXUE LU ${ }^{1}$ and ZHIGUANG SUN ${ }^{1}$ \\ ${ }^{1}$ Department of Gastroenterology, Nanjing University of Chinese Medicine, Nanjing, Jiangsu 210000; \\ ${ }^{2}$ Department of Gastroenterology, Jiangsu Second Chinese Medicine Hospital, Nanjing, Jiangsu 210017; \\ ${ }^{3}$ Department of Medical Oncology, The Second People's Hospital of Huai'an, Huaian, Jiangsu 223001, P.R. China
}

Received October 26, 2020; Accepted February 17, 2021

DOI: $10.3892 / \mathrm{etm} .2021 .10120$

\begin{abstract}
High blood glucose commonly occurs in patients with diabetes mellitus, but little is known of its effects on intestinal epithelial cells, or its associated mechanisms of action therein. In the present study, intestinal epithelial cells were assigned to five groups: i) The normal glucose (NG) group, incubated in $5.0 \mathrm{mmol} / \mathrm{l}$ glucose; ii) the constant high glucose (CHG) group, treated with $25.0 \mathrm{mmol} / \mathrm{l}$ glucose; iii) the intermittent high glucose (IHG) group, treated with alternating doses of 5.0 and $25.0 \mathrm{mmol} / \mathrm{l}$ glucose every $8 \mathrm{~h}$; iv) the mannose group, cultured in $25.0 \mathrm{mmol} / \mathrm{l}$ mannose (the osmotic control); and v) the IHG glucose + GKT137831 group, pretreated with $100 \mathrm{nmol} / \mathrm{l}$ NADPH oxidase 4 (NOX4) inhibitor, GKT137831, and then exposed to IHG. TNF- $\alpha$, IL-1 and IL-6 levels were quantified using ELISA kits. Intestinal epithelial cell apoptosis was assessed by flow cytometry and oxidative stress was evaluated by reactive oxygen species (ROS) and malondialdehyde (MDA) detection. The expression levels of proteins associated with apoptosis and involved in the signal transduction of Janus kinase (JAK)/STAT3 pathway were assessed using western blot analysis. The results indicated that NOX4 expression was significantly higher in the CHG group than in the NG group $(\mathrm{P}<0.01)$, but lower than in the IHG group $(\mathrm{P}<0.001)$. The IHG group exhibited apoptosis and oxidative stress accompanied by the most significant increase in MDA, ROS and inflammatory cytokine levels $(\mathrm{P}<0.001)$, which was followed by that of the CHG group. Additionally, the IHG group exhibited reduced
\end{abstract}

Correspondence to: Dr Zhiguang Sun, Department of Gastroenterology, Nanjing University of Chinese Medicine, 138 Xianlin Avenue, Qixia, Nanjing, Jiangsu 210000, P.R. China E-mail: sunzhiguang135@163.com

"Contributed equally

Key words: acute blood glucose fluctuation, intestinal epithelial cells, NADPH oxidase 4, reactive oxygen species, inflammation, Janus kinase/STAT3 pathway
Bcl-2, as well as enhanced Bax and cleaved caspase- 3 levels compared with the $\mathrm{CHG}$ group $(\mathrm{P}<0.001)$. Furthermore, the level of phosphorylated (p-)JAK/p-STAT3 was increased to a greater extent in the IHG group than in the CHG group $(\mathrm{P}<0.001)$. In conclusion, the findings of the present study indicated that $\mathrm{CHG}$ may trigger intestinal epithelial cell apoptosis and inflammation through the NOX4/ROS/JAK/STAT3 pathway, which may be aggravated by acute glucose fluctuation.

\section{Introduction}

Diabetes mellitus (DM) is a multi-systemic metabolic disease that is primarily characterized by hyperglycemia (1). Constant hyperglycemia can result in a series of chronic complications (2), with the gastrointestinal tract being one of the target organs (2-4). As one of the most common complications of $\mathrm{DM}$, diabetic enteropathy has attracted increasing attention; $10-20 \%$ of patients experience gastrointestinal symptoms that primarily manifest as gastrointestinal dysfunction, such as abdominal distension, intractable diarrhea and malabsorption, which has a negative impact on health-related quality of life (5). However, to date, little is known of the effects of high glucose conditions on intestinal epithelial cells.

In 1986, Mooradian et al (6) first reported that patients with DM experienced increased intestinal permeability. Subsequently, other studies have revealed that intestinal permeability is increased in patients at different stages of $\mathrm{DM}$, although it is the most obvious in the early stages of disease (7). Furthermore, Neu et al (8) discovered morphological and structural changes to intestinal cells in DM. Over the past decade, studies on the diabetic intestinal epithelium have largely focused on damage to the gut tissue from advanced glycation end-products, impaired myenteric nerve plexus function due to autonomic neuropathy and fibrosis of the intestinal muscular layers (9). However, the pathophysiological mechanisms of action underlying diabetic intestinal epithelium alterations remain to be fully elucidated.

Baynes et al have demonstrated that constant high blood glucose can directly or indirectly trigger apoptosis-associated pathways through oxidative stress or the induction of inflammation (10). Reactive oxygen species (ROS) are generated by 
several enzymes, such as NADPH oxidase (NOX), xanthine oxidase, endothelial nitric oxide synthase and enzymes of the mitochondrial electron transport system (11). NOX is reportedly the primary source of cellular ROS, including in epithelial cells (12). Emerging evidence has indicated that ROS generation can be blocked by NOX inhibitors, which has been proposed as a promising antioxidant therapy against DM $(13,14)$. Therefore in the present study, the NOX4 inhibitor, GKT137831 was used to inhibit NOX (15). The Janus kinase (JAK)/STAT3 pathway is an important intracellular signaling pathway, which plays a prominent role in inflammation and cell survival (16). This pathway can be activated by various different cytokines, such as TNF- $\alpha$, IL-1, IL- 6 and growth factors, which are involved in cell proliferation, apoptosis and migration (17). However, whether constant hyperglycemia induces intestinal epithelial cell apoptosis via the NOX4/ROS/JAK/STAT3 signaling pathway remains unclear.

It has been demonstrated that acute glucose fluctuation plays an important role in the occurrence and development of DM-associated complications (18). Whether in patients with DM or healthy subjects, acute blood glucose fluctuations may exert deleterious effects on vascular endothelial cells (19). The aim of the present study was to elucidate whether unstable hyperglycemia or glucose fluctuations promote cell apoptosis. Rat small intestinal epithelial cells (IEC-6) were used to establish an in vitro diabetic model and cellular alterations (and their underlying molecular mechanisms of action) were investigated under high glucose conditions.

\section{Materials and methods}

Cell lines, cell culture and treatment. IEC-6 cells were purchased from the American Type Culture Collection (cat. no. CRL-1592) and cultured in DMEM supplemented with $10 \%$ fetal bovine serum (HyClone; Cytiva), $100 \mathrm{U} / \mathrm{ml}$ penicillin (Sigma-Aldrich; Merck KGaA) and $100 \mathrm{mg} / \mathrm{ml}$ streptomycin (Sigma-Aldrich; Merck KGaA). The cells were maintained in a humidified incubator at $37^{\circ} \mathrm{C}$ in an atmosphere containing $5 \% \mathrm{CO}_{2}$, and the culture medium was replaced every 2 days.

Cells were assigned to five groups and then exposed to various concentrations of glucose as follows: i) Normal group (NG), $5 \mathrm{mmol}$; constant high glucose (CHG) group, $25 \mathrm{mmol}$; ii) intermittent high glucose (IHG) group, alternating between 5.0 and $25.0 \mathrm{mmol} / \mathrm{l}$ every $8 \mathrm{~h}$; iii) IHG + GKT137831 group, pretreated with $100 \mathrm{nmol} / \mathrm{l} \mathrm{GKT137831;}$ and finally, iv) the osmotic control group, which was treated with $25 \mathrm{mmol} / 1$ mannose.

Western blotting. Western blotting was conducted according to previously described methods (20). IEC-6 cells were lysed in RIPA buffer (Beyotime Institute of Biotechnology) containing protease inhibitors (Sigma-Aldrich; Merck KGaA), phosphatase inhibitors (Sigma-Aldrich; Merck KGaA) and PMSF (Beyotime Institute of Biotechnology). Total protein was extracted and quantified using a bicinchoninic acid kit (Beyotime Institute of Biotechnology). Lysed protein $(20 \mu \mathrm{g})$ was separated by $10 \%$ SDS-PAGE and then transferred to PVDF membranes (Stratagene; Agilent Technologies, Inc.). The membranes containing proteins were blocked for $1.5 \mathrm{~h}$ in $5 \% \mathrm{BSA}$ at room temperature. Subsequently, the membranes were incubated with primary antibodies against NOX4 (cat. no. ab109225; Abcam), Bcl-2 (cat. no. D17C4; Cell Signaling Technology, Inc.), Bax (cat. no. 2772; Cell Signaling Technology, Inc.), cleaved caspase-3 (cat. no. 9664; Cell Signaling Technology, Inc.), phosphorylated (p-)JAK (cat. no. 66245; Cell Signaling Technology, Inc.), p-STAT3 (cat. no. 9145; Cell Signaling Technology, Inc.), JAK (cat. no. 3344; Cell Signaling Technology, Inc.), STAT3 (cat. no. 9139; Cell Signaling Technology, Inc.) and GAPDH (cat. no. A19056; ABclonal, Biotech Co., Ltd.) overnight at $4^{\circ} \mathrm{C}$. The aforementioned antibodies were diluted at ratio of 1:1,000. Membranes were then incubated with HRP-conjugated goat-anti-rabbit IgG (1:10,000; cat. no. AS014; ABclonal, Biotech Co., Ltd.) and HRP-conjugated goat-anti-mouse IgG secondary antibodies (1:10,000; cat. no. AS003; ABclonal, Biotech Co., Ltd.) for $2 \mathrm{~h}$ at room temperature. Finally, Protein bands were visualized by enhanced chemiluminescence detection (Amersham; Cytiva) and quantified by Image J software (version 1.46, National Institutes of Health). Target protein expression is presented relative to that of GAPDH.

Immunocytofluorescence assay. After fixing with $4 \%$ formaldehyde (Sigma-Aldrich; Merck KGaA) at $4^{\circ} \mathrm{C}$ for $30 \mathrm{~min}$, the cells were permeabilized with $0.1 \%$ Triton $\mathrm{X}-100$ in PBS and then blocked with $10 \%$ normal goat serum (Invitrogen; Thermo Fisher Scientific, Inc.) for $1 \mathrm{~h}$ at room temperature. The cells were subsequently incubated with primary antibodies against NOX4 (1:100; cat. no. PA5-72816; Thermo Fisher Scientific, Inc.) at $4^{\circ} \mathrm{C}$ overnight, followed by FITC-conjugated anti-rabbit IgG secondary antibody (1:100) for $1 \mathrm{~h}$ at room temperature (cat. no. A30008; Invitrogen; Thermo Fisher Scientific, Inc.). Fluorescence was assessed by fluorescence microscopy (magnification, x400; Leica Microsystems $\mathrm{GmbH}$ ). Quantification of fluorescence intensity or positive cell/nuclear ratios was performed using ImageJ version 1.47 (National Institutes of Health). The results are presented as the mean \pm standard error of the mean (SEM) from $\geq 3$ independent experiments.

ELISA. The samples were centrifuged at $1,500 \mathrm{xg}$ for $15 \mathrm{~min}$ at $4^{\circ} \mathrm{C}$ to obtain the cell supernatant. The expression levels of TNF- $\alpha$ (cat. no. ER006-96), IL-1 (cat. no. ER008-96) and IL-6 (cat. no. ER003-96) in the cell supernatant were determined using ELISA kits (Shanghai ExCell Biology, Inc.) according to the manufacturers' protocols. The cell supernatant was evaluated using a multiclan spectrum spectrophotometer (Thermo Fisher Scientific, Inc.).

Detection of intracellular ROS generation. Following appropriate group treatments, the cells were harvested and incubated with the fluorescent probe, 2',7'-dichlorodihydrofluorescein diacetate $(0.1 \%)$, at $37^{\circ} \mathrm{C}$ for $30 \mathrm{~min}$. After resuspension, the fluorescence intensity was analyzed using a microplate reader (Thermo Fisher Scientific, Inc.).

Detection of malondialdehyde (MDA) levels. The generation of MDA in the cells was assessed using an MDA ELISA assay kit (cat. no. S0131S; Beyotime Institute of Biotechnology). The cells were homogenized with PBS and the MDA detection working solution was mixed with the cell homogenate. Subsequently, the mixed solution was heated at $100^{\circ} \mathrm{C}$ for 


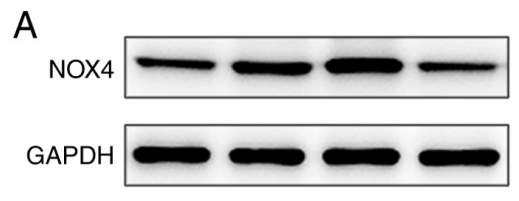

B
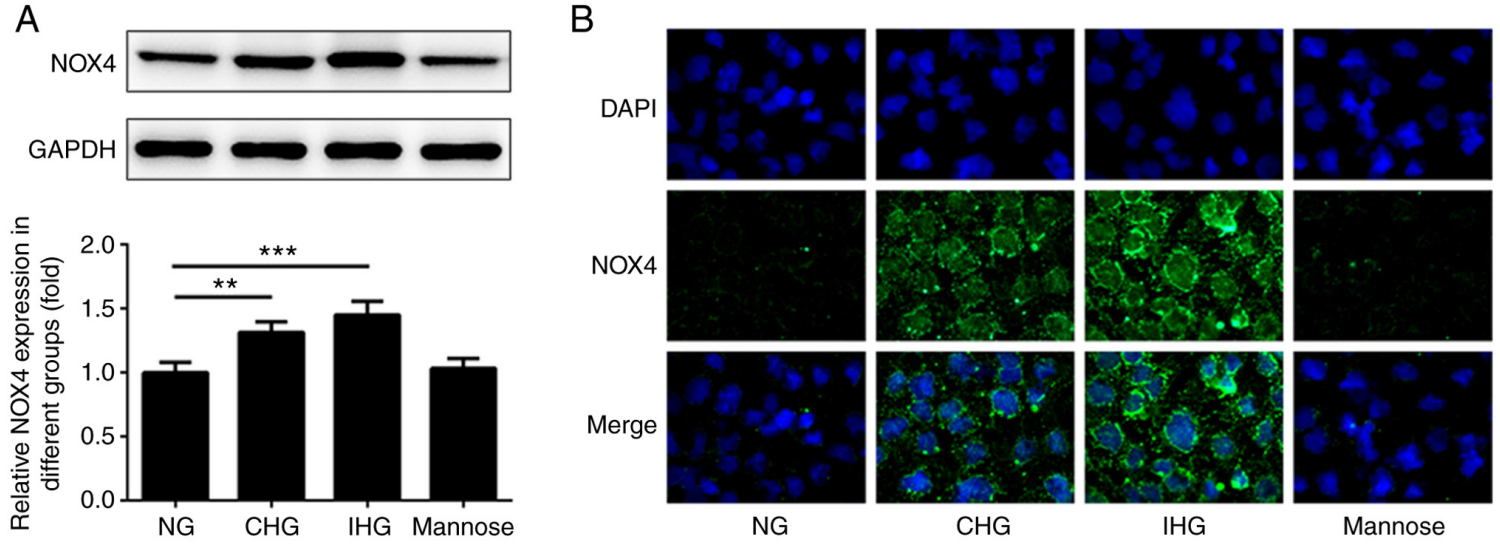

Figure 1. NOX4 expression in intestinal epithelial cells among the various treatment groups. (A) NOX4 protein expression levels in intestinal epithelial cells were assessed using western blot analysis. (B) Representative immunofluorescence staining images of NOX4 expression (green) in intestinal epithelial cells; nuclei were stained with DAPI (blue); magnification, $\mathrm{x} 400$. $^{* *} \mathrm{P}<0.01$ and ${ }^{* * * *} \mathrm{P}<0.001$; $\mathrm{n} \geq 3$. CHG, constant high glucose; IHG, intermittent high glucose; NG, normal glucose; NOX4, NADPH oxidase 4.

$15 \mathrm{~min}$. The absorbance was then measured at $532 \mathrm{~nm}$ using a microplate reader (Thermo Fisher Scientific, Inc.).

Apoptosis analysis. The apoptotic rates of small intestinal epithelial cells were assessed by flow cytometry, following a series of previously described procedures (21). The cells were harvested and stained with Annexin V-fluorescein isothiocyanate (FITC) and propidium iodide using the Annexin VFITC Apoptosis Detection kit I (BD Biosciences), and were then incubated in the dark for $15 \mathrm{~min}$ at room temperature. The cells were subsequently analyzed within $1 \mathrm{~h}$ using BD FACSVerse ${ }^{\mathrm{TM}}$ flow cytometer (BD Biosciences) and the Flow $\mathrm{Jo}^{\mathrm{TM}} \mathrm{VX} 10$ software (FlowJo LLC) was used to analyze the data.

Statistical analysis. All data were analyzed by one-way analysis of variance followed by Tukey's post hoc test, which was conducted using GraphPad 6.0 (GraphPad Software, Inc.) Data are presented as the means \pm SEM and $\mathrm{P}<0.05$ was considered to indicate a statistically significant difference. All experiments were repeated at least three times.

\section{Results}

Comparison of $\mathrm{NOX} 4$ expression between the various treatment groups. Changes in NOX4 expression in intestinal epithelial cells were detected under fluctuating glucose concentrations using western blotting and immunocytofluorescence staining. As indicated in Fig. 1A-B, the expression levels of NOX4 in both the CHG and IHG groups were significantly increased compared with that in the NG group, and to a greater extent in the IHG group than in the CHG group. Moreover, there were no significant differences in NOX4 expression levels between the mannose group and the NG group at $24 \mathrm{~h}$ post-treatment. In addition, the fluorescence intensity (green stain) of NOX4 in the CHG and IHG groups was increased compared with the NG group. NOX4 expression in the IHC group was also higher than the $\mathrm{CHG}$ group. However, there was no significant difference between the mannose group and the NG group.

Comparison of inflammatory cytokine expression levels between groups. Compared with the NG group, the levels of inflammatory cytokines (TNF- $\alpha$, IL-1 and IL-6) in the CHG and IHG groups were markedly increased, and the levels in the CHG group where lower than those in the IHG group. Notably, the levels of TNF- $\alpha$, IL-1 and IL- 6 in the IHG group were decreased following pretreatment with GKT137831, but were still higher than those in the NG group. In addition, there was no significant difference between the mannose group and the NG group (Fig. 2A-C).

Expression of oxidative stress-associated biomarkers. Oxidative stress is reported to play an important role in intestinal epithelial cell injury under high glucose conditions. Compared with the NG group, the levels of oxidative stress biomarkers [ROS and MDA] were increased in the CHG and IHG groups, with the highest levels found in the IHG group (Fig. 3A and B). Notably, the levels of ROS and MDA activity in the IHG + GKT137831 group were lower than those in the IHG group, but higher than those in the NG group. There was no significant difference between the mannose group and the NG group. These results suggested that blood glucose fluctuation exacerbates oxidative stress in intestinal epithelial cells.

Blood glucose fluctuation induces apoptosis in intestinal epithelial cells. Flow cytometry was used to detect intestinal epithelial cell apoptosis following blood glucose fluctuation. As shown in Fig. 4A, apoptosis in the IHG and CHG groups was significantly higher than that in the NG group and was at its highest level in the IHG group; there was no significant difference between the mannose control and NG groups. In addition, compared with the IHG group, the level of apoptosis in the IHG + GKT137831 group was significantly decreased, but remained higher than that in the NG group. These results indicated that blood glucose fluctuation aggravates intestinal epithelial cell apoptosis.

Effects of blood glucose fluctuation on the expression of apoptosis-associated proteins. Compared with the NG group, the expression levels of Bax and cleaved caspase 3/caspase 3 was significantly increased in the CHG and IHG groups, while the expression levels of Bcl-2 was decreased (Fig. 4B). Furthermore, Bax and cleaved caspase- 3 expression levels were higher in the 
A

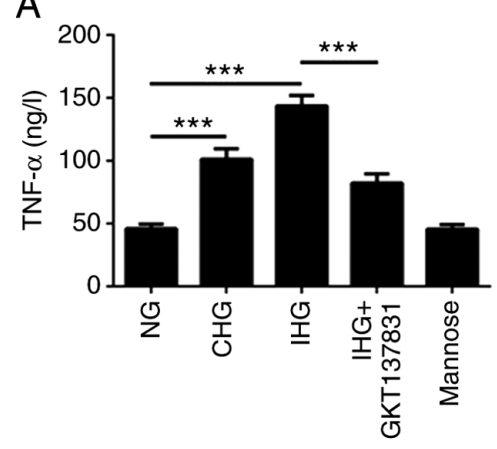

B

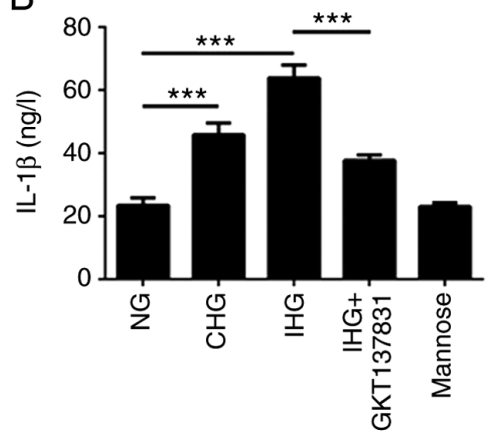

C

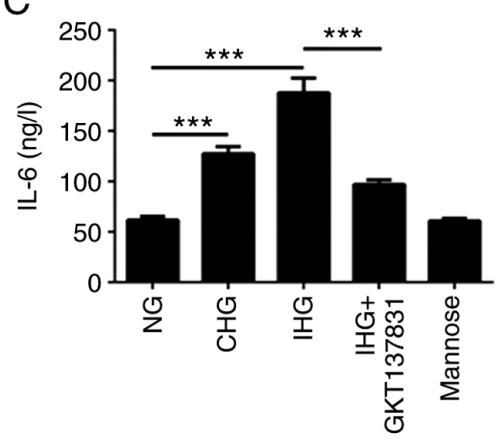

Figure 2. Blood glucose fluctuation induces inflammation in intestinal epithelial cells. Plasma inflammatory cytokine levels, namely (A) TNF- $\alpha$, (B) IL-1 $\beta$ and (C) IL-6, in intestinal epithelial cells between the various treatment groups. ${ }^{* * *} \mathrm{P}<0.001, \mathrm{n} \geq 3$. CHG, constant high glucose; IHG, intermittent high glucose; NG, normal glucose.

A

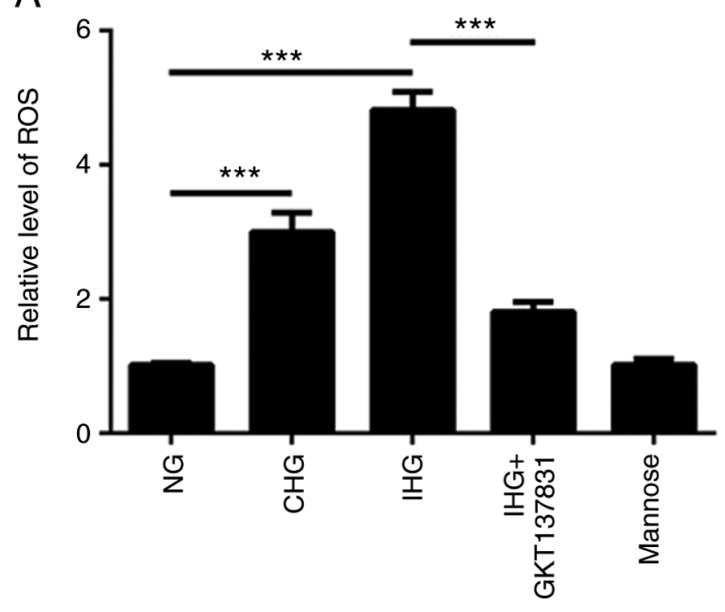

B

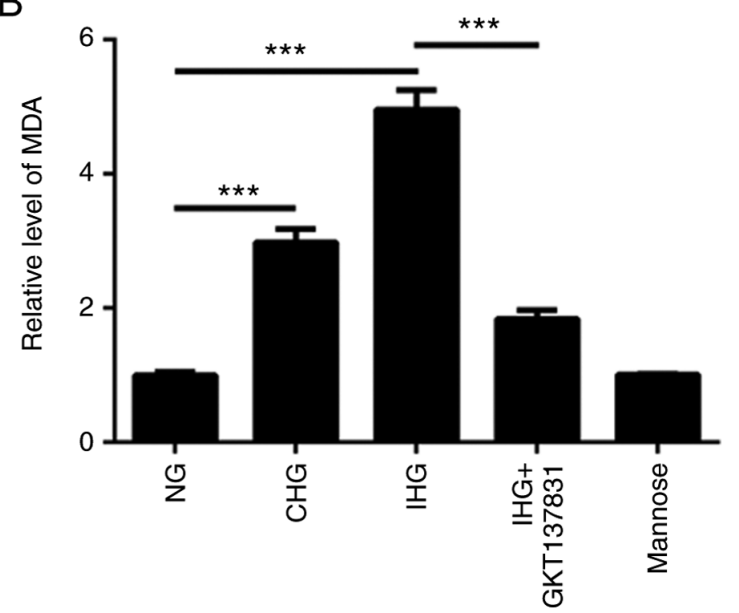

Figure 3. Blood glucose fluctuation induces oxidative stress in intestinal epithelial cells. (A and B) Expression of biomarkers of oxidation between groups. ${ }^{* * *} \mathrm{P}<0.001$ vs. the NG and IHG groups; $\mathrm{n} \geq 3$. CHG, constant high glucose; IHG, intermittent high glucose; MDA, malondialdehyde; NG, normal glucose; ROS, reactive oxygen species.

IHG group than in the CHG group. In addition, compared with the IHG group, the levels of Bax and cleaved caspase- 3 were significantly decreased in the IHG + GKT137831 group, but higher than those in the NG group, and the IHG + GKT137831 group exhibited significantly increased Bcl-2 expression compared with the IHG group. There were no significant differences in the levels of Bax, cleaved caspase- 3 and $\mathrm{Bcl}-2$ between the mannose group and the NG group.

Role of the JAK/STAT3 pathway in inflammation and cell survival. To determine the effects of glucose fluctuation on the JAK/STAT3 pathway in intestinal epithelial cells, p-JAK and p-STAT3 levels were detected in IEC- 6 cells exposed to fluctuating glucose concentrations. As shown in Fig. 5, the ratios of p-JAK/JAK and p-STAT3/STAT3 in the CHG and IHG groups were significantly higher than those in the NG group, though the increase was more pronounced in the IHG group. Moreover, compared with the IHG group, the ratios of p-JAK/JAK and p-STAT3/STAT3 in the IHG + GKT137831 group were significantly decreased, although they remained higher significantly than those in the NG group. There were no significant differences in the levels of p-JAK and p-STAT3 between the mannose group and the NG group. These results indicated that glucose fluctuation accelerates intestinal epithelial cell apoptosis and that this is associated with increased phosphorylation of JAK and STAT3.

\section{Discussion}

To the best of our knowledge, the present study is the first to investigate the changes in intestinal epithelial cells under high glucose conditions, as well as the associated underlying pathophysiological mechanisms. When investigating the influence of acute glucose fluctuations on cells, a high glucose concentration is consider to be $25 \mathrm{mmol}(22,23)$. At this concentration, acute glucose fluctuations can induce inflammation and apoptosis $(22,23)$. As such, the present study used this concentration for all experiments. The following results were noted: i) $\mathrm{CHG}$ increased the expression of NOX4, ROS, apoptosis-associated proteins and inflammatory factors in intestinal epithelial cells, as well as, ultimately, the number of apoptotic cells, which was exacerbated by acute glucose fluctuation; ii) a persistent high glucose concentration upregulated the phosphorylation levels of JAK and STAT3, which was further increased by acute glucose fluctuation, In parallel with the points outlined in i); and iii) the levels of NOX4, ROS, apoptosis-associated proteins, 
A
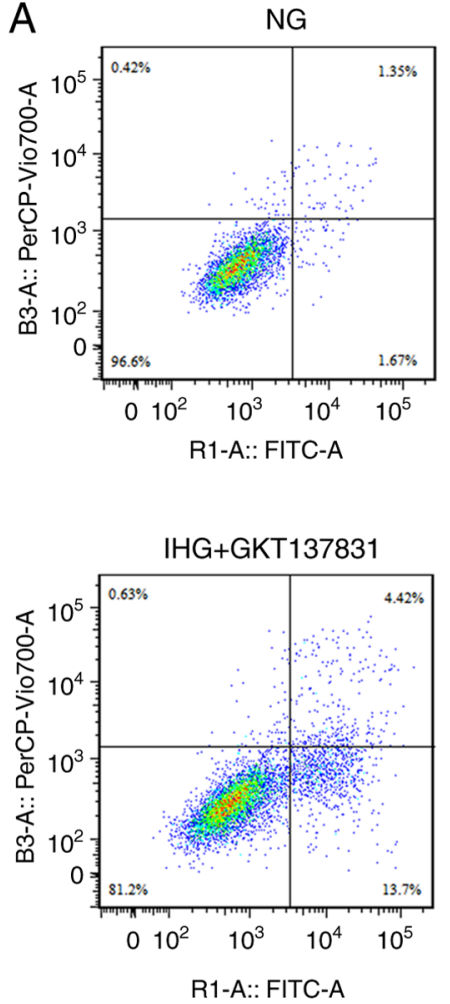

B

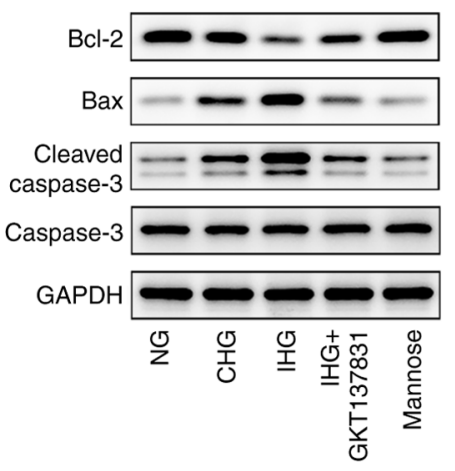

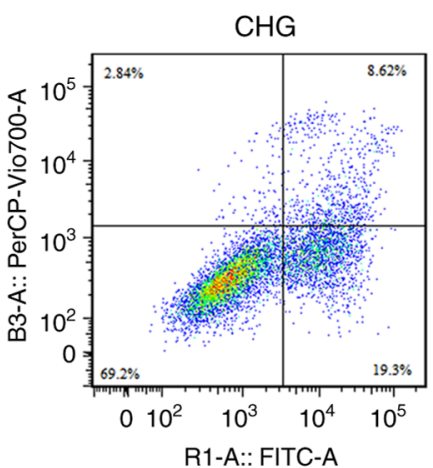
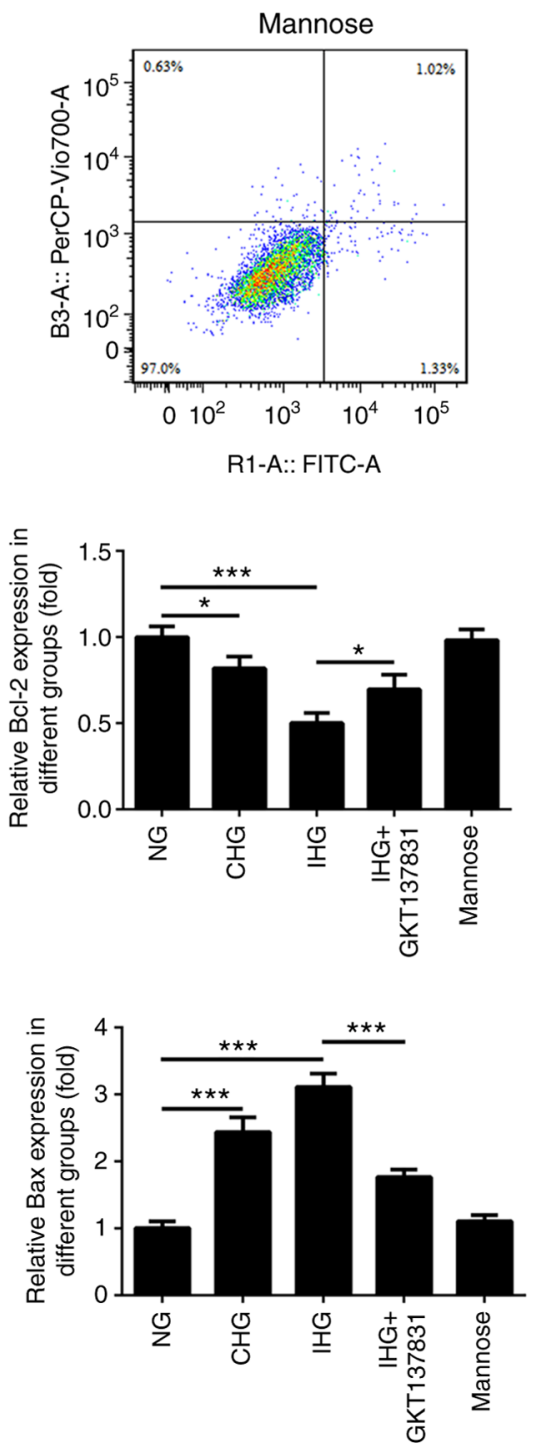
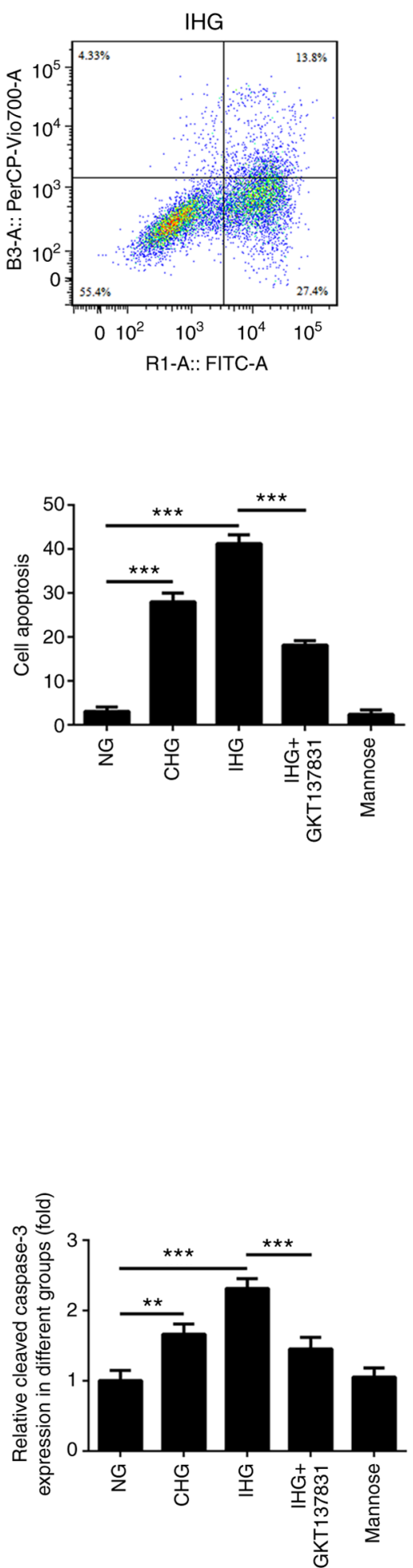

Figure 4. Blood glucose fluctuation induces apoptosis in intestinal epithelial cells. (A) Results of flow cytometry analysis on the extent of apoptosis between the various treatment groups. (B) Western blot analysis of Bcl-2, Bax, cleaved caspase-3 and caspase-3 protein expression levels in intestinal epithelial cells. ${ }^{*} \mathrm{P}<0.1,{ }^{* *} \mathrm{P}<0.01$ and ${ }^{* * *} \mathrm{P}<0.001 ; \mathrm{n} \geq 3$. CHG, constant high glucose; IHG, intermittent high glucose; NG, normal glucose.

inflammatory factors, p-JAK and p-STAT3 were markedly downregulated following NOX inhibition.

Furthermore, the number of apoptotic intestinal epithelial cells was higher in the $\mathrm{CHG}$ group compared with those in the NG group, suggesting that apoptosis was induced by $\mathrm{CHG}$. Therefore, it was hypothesized that the hyperglycemia-induced apoptosis of intestinal epithelial cells may account for the occurrence and development of diabetic enteropathy, which is supported by the results of previous studies. In 2003, Quagliaro et al (24) reported that high glucose triggered apoptosis in human umbilical vein endothelial cells. Such effects have also been observed in vascular endothelial cells, mesangial cells, myocardial cells and pancreatic islets $(21,24,25)$. However, the results of previous studies investigating the 

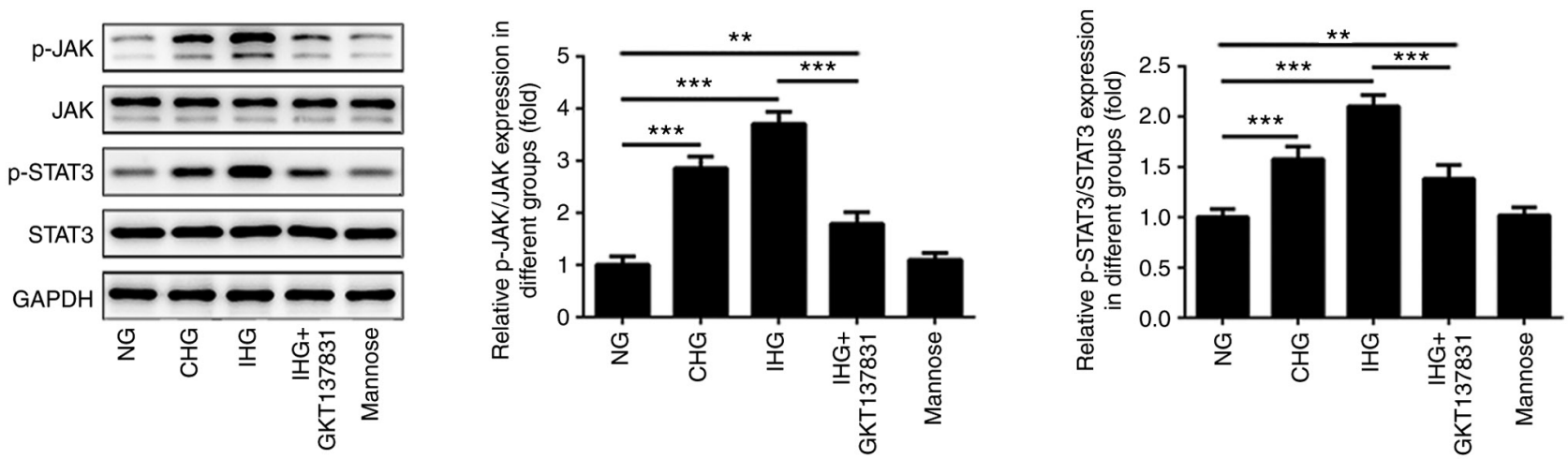

Figure 5. Glucose fluctuation promotes the phosphorylation of JAK and STAT3 in intestinal epithelial cells. Western blot analysis of p-JAK, JAK, p-STAT3 and STAT3 levels in intestinal epithelial cells between the various treatment groups. ${ }^{* * *} \mathrm{P}<0.01$ and ${ }^{* * * *} \mathrm{P}<0.001 ; \mathrm{n} \geq 3$. CHG, constant high glucose; IHG, intermittent high glucose; JAK, janus kinase; NG, normal glucose; p-, phosphorylated.

changes in intestinal epithelial cells under constant hyperglycemia have been inconsistent. Other studies have indicated that the proliferation of various cell types, such as myocardial, mammary and placental cells, are enhanced under hyperglycemic and hyperinsulinemic conditions (26-28). An animal study also revealed that the intestinal epithelium of rats with hyperglycemia displayed longer villi, increased wet weight, increased cellular proliferation and hypertrophy within the crypts (29). In addition, patients with DM reportedly are at an increased risk of developing colorectal cancer (30) and diabetic rats are more susceptible to colorectal tumorigenesis (31). At present, further investigation is required to fully elucidate the association between intestinal epithelial cell injury and CHG.

Hyperglycemia is common in patients with DM, which can result in oxidative stress and may be responsible for the emergence and development of complications (32). Increasing clinical evidence has demonstrated that DM-related complications are more pronounced in association with peak or post-meal, rather than average blood glucose levels (25). Furthermore, in vivo studies have shown that apoptosis, oxidative stress and pro-inflammatory cytokine release are enhanced by acute blood glucose fluctuation in human umbilical vein endothelial cells, vascular endothelial cells, mesangial cells, myocardial cells and pancreatic islets (33-35), which has been further confirmed by a number of in vitro studies $(36,37)$.

The correlation between blood glucose fluctuations and diabetes complications has received extensive attention in recent years (38). Compared to persistent hyperglycemia, fluctuant hyperglycemia has a greater potential to increase microvascular lesions and the risk of cardiovascular death $(18,39)$, but the specific mechanism of action remains unclear. One possible explanation is that oxidative stress may play an important role in cell damage caused by blood glucose fluctuations. Studies have shown that the levels of oxidative stress have no significant correlation with fasting blood glucose, but have a significant correlation with expression of markers of acute glucose fluctuation (18). This suggested that oxidative stress increases more as a result of larger glucose fluctuations (18). Additionally, as a result of continuous hyperglycemia, certain compensation or feedback reactions are induced in cells to compensate for the constant stimulation (24). However, in the intermittent hyperglycemic state, it is speculated that such an adaptative reaction is reduced or does not function properly (24). However, further researches are required to confirm this speculation.

Oxidative stress promotes redox imbalance and a marked increase in the generation of ROS and inflammatory cytokines, which ultimately results in enhanced apoptosis (40). Studies have demonstrated that oxidative stress can cause inflammation, but no inflammation occurs during cell apoptosis $(41,42)$. Inflammation will further aggravate oxidative stress and IL-6 can play an important role through the JAK/STAT3 pathway (43). In the present study, oxidative stress was caused by glucose fluctuation. Therefore, it is believed that the release of inflammatory factors is partly caused by this glucose fluctuation. Studies have also shown that NOX4 may lead to necroptosis and thus release inflammatory factors (44). Whether acute glucose fluctuations lead to necroptosis requires further verification in intestinal epithelial cells.

The NOX family proteins are multicomponent enzymes that are the key source of ROS generation in various cell types, as well as in rodent diabetic models (45). The NOX family is composed of seven members, including NOX1 (mainly in colon tissues), NOX2 (primarily expressed in phagocytes), NOX3 (predominantly in the inner ear), NOX4, NOX5 (mainly expressed in lymphoid tissues), Duxo1 and Duxo2 (both mainly in the thyroid and bronchus) (46). Of note, NOX4 is also expressed in intestinal tissue $(47,48)$. NOX4 has also been shown to play a potentially important role in diabetes and its complications (49). In addition, the activation of NOX can also cause inflammation and apoptosis (45). Therefore, the present study examined the role of NOX4 in inflammation and apoptosis induced by acute glucose fluctuation in intestinal epithelial cells.

The results of the present study confirmed that the expression of NOX4, ROS, apoptosis-associated proteins and inflammatory factors (as well as the number of apoptotic cells) was raised in the IHG group compared with that in the CHG group, indicating that acute glucose fluctuation exacerbates intestine epithelial cell apoptosis. Since the signal transduction cascades involved in DM are associated with the activation of various molecules (such as transcription factors, cytokines, hormones and protein kinases), the present study aimed to investigate the underlying molecular mechanisms of action involved in the effects of acute blood fluctuation. The results revealed that high glucose upregulated the levels of NOX4, ROS, p-JAK and 
p-STAT3, which was further exacerbated by acute glucose fluctuation. This effect was suppressed by a NOX inhibitor, suggesting that acute high glucose enhances intestinal epithelial cell apoptosis by activating the NOX4/ROS/JAK/STAT3 signaling pathway. These results were consistent with those of previous reports $(50,51)$. The JAK/STAT3 signaling pathway is also reportedly implicated in the pathophysiology behind DM (52). Studies have demonstrated that, in diabetic rodents, the apoptosis and abnormal proliferation of vascular endothelial cells, mesangial cells, myocardial cells and sensory neurons was promoted by JAK/STAT3 pathway activation, leading to organ injury and deterioration (52-55). This organ damage was suppressed and/or ameliorated by STAT3-knockdown or JAK inhibition $(56,57)$. However, several studies have also shown that activation of the JAK/STAT3 pathway protects against myocardial ischemia-reperfusion injury, myocardial cell apoptosis and inflammation $(58,59)$. Differences between various animal models and the course of the disease may explain the effective differences resulting from JAK/STAT3 pathway activation.

In conclusion, the results of the present study suggested that $\mathrm{CHG}$ triggers intestinal epithelial cell apoptosis through the NOX4/ROS/JAK/STAT3 signaling pathway, which is subsequently enhanced by acute glucose fluctuation. The current study has demonstrated that acute glucose fluctuation inflicts greater damage to intestinal epithelial cells, further emphasizing the importance of glucose control.

\section{Acknowledgements}

Not applicable.

\section{Funding}

This work was supported by Postgraduate Research and Practice Innovation Program of Jiangsu Province (grant no. KYCX20 1480).

\section{Availability of data and materials}

The datasets used and/or analyzed during the current study are available from the corresponding author on reasonable request.

\section{Authors' contributions}

$\mathrm{ZS}$ and $\mathrm{BC}$ designed the study. $\mathrm{BC}$ and $\mathrm{YJ}$ performed the literature search and selection. BC, YJ and DL performed the experiment and analyzed the data. BC and YJ drafted the manuscript. All authors revised and approved the final manuscript. ZS and BC confirm the authenticity of all the raw data.

\section{Ethics approval and consent to participate}

Not applicable.

\section{Patient consent for publication}

Not applicable.

\section{Competing interests}

The authors declare that they have no competing interests.

\section{References}

1. Wilson PWF, D'Agostino RB, Helen P, Lisa S and Meigs JB: Metabolic syndrome as a precursor of cardiovascular disease and type 2 diabetes mellitus. Circulation 112: 3066-3072, 2005.

2. Brownlee M: Biochemistry and molecular cell biology of diabetic complications. Nature 414: 813-820, 2001.

3. Papatheodorou K, Banach M, Bekiari E, Rizzo M and Edmonds M: Complications of diabetes 2017. J Diabetes Res 2018: 3086167, 2018.

4. Snelson M, de Pasquale C, Ekinci EI and Coughlan MT: Gut microbiome, prebiotics, intestinal permeability and diabetes complications. Best Pract Res Clin Endocrinol Metab, 2021 (Ahead of print).

5. Talley NJ, Young L, Bytzer P, Hammer J, Leemon M, Jones M and Horowitz M: Impact of chronic gastrointestinal symptoms in diabetes mellitus on health-related quality of life. Am J Gastroenterol 96: 71-76, 2001.

6. Mooradian AD, Morley JE, Levine AS, Prigge WF and Gebhard RL: Abnormal intestinal permeability to sugars in diabetes mellitus. Diabetologia 29: 221-224, 1986.

7. Meddings JB, Jarand J, Urbanski SJ, Hardin J and Gall DG: Increased gastrointestinal permeability is an early lesion in the spontaneously diabetic BB rat. Am J Physiol 276: 951-957, 1999.

8. Neu J, Reverte CM, Mackey AD, Liboni K, Tuhacek-Tenace LM, Hatch M, Li N, Caicedo RA, Schatz DA and Atkinson M: Changes in intestinal morphology and permeability in the biobreeding rat before the onset of type 1 diabetes. J Pediatr Gastroenterol Nutr 40: 589-595, 2005.

9. Chocarro-Calvo A, García-Martínez JM, Ardila-González S, De la Vieja A and García-Jiménez C: Glucose-induced $\beta$-catenin acetylation enhances Wnt signaling in cancer. Mol Cell 49: 474-486, 2013

10. Baynes JW and Thorpe SR: Role of oxidative stress in diabetic complications: A new perspective on an old paradigm. Diabetes 43: $1-9,1999$.

11. Babior BM: NADPH oxidase. Curr Opin Immunol 16: 42-47, 2004.

12. Ago T, Kuroda J, Kamouchi M, Sadoshima J and Kitazono T: Pathophysiological roles of NADPH oxidase/nox family proteins in the vascular system. Review and perspective. Circ J 75: 1791-1800, 2011

13. Asaba K, Tojo A, Onozato ML, Goto A, Quinn MT, Fujita T and Wilcox CS: Effects of NADPH oxidase inhibitor in diabetic nephropathy. Kidney Int 67: 1890-1898, 2005.

14. Roe ND, Thomas DP and Ren J: Inhibition of NADPH oxidase alleviates experimental diabetes-induced myocardial contractile dysfunction. Diabetes Obes Metab 13: 465-473, 2011.

15. Teixeira G, Szyndralewiez C, Molango S, Carnesecchi S, Heitz F, Wiesel P and Wood JM: Therapeutic potential of NADPH oxidase 1/4 inhibitors. Br J Pharmacol 174: 1667-1669, 2017.

16. Wang JW, Pan YB, Cao YQ, Wang C, Jiang WD, Zhai WF and Lu JG: Loganin alleviates LPS-activated intestinal epithelial inflammation by regulating TLR4/NF- $\mathrm{BB}$ and JAK/STAT3 signaling pathways. Kaohsiung J Med Sci 36: 257-264, 2020.

17. Hirano T, Ishihara KM and Hibi M: Roles of STAT3 in mediating the cell growth, differentiation and survival signals relayed through the IL-6 family of cytokine receptors. Oncogene 19: 2548-2556, 2000.

18. Monnier L, Mas E, Ginet C, Michel F, Villon L, Cristol JP and Colette C: Activation of oxidative stress by acute glucose fluctuations compared with sustained chronic hyperglycemia in patients with type 2 diabetes. JAMA 295: 1681-1687, 2006.

19. Ceriello A, Esposit K, Piconi L, Ihnat MA, Thorpe JE, Testa R, Boemi M and Giugliano D: Oscillating glucose is more deleterious to endothelial function and oxidative stress than mean glucose in normal and type 2 diabetic patients. Diabetes 57: 1349-1354, 2008.

20. Lee MK, Kim IH, Choi YH and Nam TJ: A peptide from Porphyra yezoensisstimulates the proliferation of IEC- 6 cells by activating the insulin-like growth factor I receptor signaling pathway. Int J Mol Med 35: 533-538, 2015.

21. Shen JT, Li YS, Xia ZQ, Wen SH, Yao X, Yang WJ, Li C and Liu KX: Remifentanil preconditioning protects the small intestine against ischemia/reperfusion injury via intestinal $\delta$ - and $\mu$-opioid receptors. Surgery 159: 548-559, 2016.

22. Ying C, Wang S, Lu Y, Chen L, Mao Y, Ling H, Cheng X and Zhou X: Glucose fluctuation increased mesangial cell apoptosis related to AKT signal pathway. Arch Med Sci 15: 730-737, 2019. 
23. Hsieh CF, Liu CK, Lee CT, Yu LE and Wang JY: Acute glucose fluctuation impacts microglial activity, leading to inflammatory activation or self-degradation. Sci Rep 9: 840, 2019.

24. Quagliaro L, Piconi L, Assaloni R, Martinelli L, Motz E and Ceriello A: Intermittent high glucose enhances apoptosis related to oxidative stress in human umbilical vein endothelial cells: The Role of Protein Kinase C and NAD(P)H-Oxidase Activation. Diabetes 52: 2795-2804, 2003.

25. Cho JH, Chang SA, Kwon HS, Choi YH, Ko SH, Moon SD, Yoo SJ, Song KH, Son HS, Kim HS, et al: Long-term effect of the Internet-based glucose monitoring system on HbAlc reduction and glucose stability: A 30-month follow-up study for diabetes management with a ubiquitous medical care system. Diabetes Care 29: 2625-2631, 2006.

26. Liu Q, Huang QX, Lou FC, Zhang L, Hou WK, Yu S, Xu H, Wang $Q$, Zhang Y and Hou WK: Effects of glucose and insulin on the H9c2 (2-1) cell proliferation may be mediated through regulating glucose transporter 4 expression. Chin Med J (Engl) 126 4037-4042, 2013.

27. Lopez R, Arumugam A, Joseph R, Monga K, Boopalan T, Agullo P, Gutierrez C, Nandy S, Subramani R, de la Rosa JM and Lakshmanaswamy R: Hyperglycemia enhances the proliferation of non-tumorigenic and malignant Mammary epithelial cells through increased leptin/IGF1R signaling and activation of AKT/mTOR. PLoS One 8: e79708, 2013.

28. Ozmen A, Unek G, Kipmen-Korgun D and Korgun ET: The PI3K/Akt and MAPK-ERK1/2 pathways are altered in STZ induced diabetic rat placentas. Histol Histopathol 29: 743-756, 2013.

29. Adachi T, Mori C, Sakurai K, Shihara N, Tsuda K and Yasuda K Morphological changes and increased sucrase and isomaltase activity in small intestines of insulin-deficient and type 2 diabetic rats. Endocr J 50: 271-279, 2003.

30. Rasool S, Kadla SA, Rasool V and Ganai BA: A comparative overview of general risk factors associated with the incidence of colorectal cancer. Tumor Biology 34: 2469-2476, 2013.

31. Hata K, Kubota M, Shimizu M, Moriwaki H, Kuno T, Tanaka T, Hara A and Hirose Y: Monosodium glutamate-induced diabetic mice are susceptible to azoxymethane-induced colon tumorigenesis. Carcinogenesis 33: 702-707, 2012.

32. King GL and Loeken MR: Hyperglycemia-induced oxidative stress in diabetic complications. Histochem Cell Biol 122: 333-338, 2004

33. Wu N, Shen H, Liu H, Wang Y, Bai Y and Han P: Acute blood glucose fluctuation enhances rat aorta endothelial cell apoptosis, oxidative stress and pro-inflammatory cytokine expression in vivo. Cardiovasc Diabetol 15: 109, 2016.

34. Sharma A, Tate M, Mathew G, Vince JE, Ritchie RH and Haan JB: Oxidative stress and NLRP3-inflammasome activity as significant drivers of diabetic cardiovascular complications: Therapeutic implications. Front Physiol 9: 114, 2018.

35. Lal MA, Brismar H, Eklöf AC and Aperia A: Role of oxidative stress in advanced glycation end product-induced mesangial cell activation. Kidney Int 61: 2006-2014, 2002.

36. Zhang W, Zhao S, Li Y, Peng G and Han P Acute blood glucose fluctuation induces myocardial apoptosis through oxidative stress and nuclear factor-kB activation. Cardiology 124: 11-17, 2013.

37. Xue B, Wang L, Zhang Z, Wang R, Xia XX, Han PP, Cao LJ, Liu YH and Sun LQ: Puerarin may protect against Schwann cell damage induced by glucose fluctuation. J Nat Med 71: 472-481, 2017.

38. Xia J, Hu S, Xu J, Hao H, Yin C and Xu D: The correlation between glucose fluctuation from self-monitored blood glucose and the major adverse cardiac events in diabetic patients with acute coronary syndrome during a 6-month follow-up by WeChat application. Clin Chem Lab Med 56: 2119-2124, 2018.

39. Glucose tolerance and mortality: Comparison of WHO and American Diabetes Association diagnostic criteria. The DECODE study group. European Diabetes Epidemiology Group. Diabetes Epidemiology: Collaborative analysis Of Diagnostic criteria in Europe. Lancet 354: 617-621, 1999.

40. Curtin JF, Donovan M and Cotter TG: Regulation and measurement of oxidative stress in apoptosis. J Immunol Methods 265: 49-72, 2002.

41. Nikoletopoulou V, Markaki M, Palikaras K and Tavernarakis N: Crosstalk between apoptosis, necrosis and autophagy. Biochim Biophys Acta 1833: 3448-3459, 2013.

42. Hussain T, Tan B, Yin Y, Blachier F, Tossou MC and Rahu N: Oxidative stress and inflammation: What Polyphenols Can Do for Us? Oxid Med Cell Longev 2016: 7432797, 2016.
43. Shen Y, Zhang Q, Huang Z, Zhu J, Qiu J, Ma W, Yang X, Ding F and Sun H: Isoquercitrin delays denervated soleus muscle atrophy by inhibiting oxidative stress and inflammation. Front Physiol 11: 988, 2020.

44. Meng XM, Ren GL, Gao L, Yang Q, Li HD, Wu WF, Huang C, Zhang L, Lv XW and Li J: NADPH oxidase 4 promotes cisplatin-induced acute kidney injury via ROS-mediated programmed cell death and inflammation. Lab Invest 98: 63-78, 2018.

45. Sedeek M, Montezano AC, Hebert RL, Gray SP, Di Marco E, Jha JC, Cooper ME, Jandeleit-Dahm K, Schiffrin EL, Wilkinson-Berka JL and Touyz RM: Oxidative stress, Nox isoforms and complications of diabetes-potential targets for novel therapies. J Cardiovasc Transl Res 5: 509-518, 2012.

46. Sedeek M, Nasrallah R, Touyz RM and Hebert RL: NADPH oxidases, reactive oxygen species, and the kidney: Friend and foe. J Am Soc Nephrol 24: 1512-1518, 2013.

47. Chu FF, Esworthy RS, Shen B, Gao Q and Doroshow JH: Dexamethasone and Tofacitinib suppress NADPH oxidase expression and alleviate very-early-onset ileocolitis in mice deficient in GSH peroxidase 1 and 2. Life Sci 239: 116884, 2019.

48. Lindquist RL, Bayat-Sarmadi J, Leben R, Niesner R and Hauser AE: NAD $(\mathrm{P}) \mathrm{H}$ oxidase activity in the small intestine is predominantly found in enterocytes, not professional phagocytes. Int J Mol Sci 19: 1365, 2018.

49. Yan J, Wang C, Jin Y, Meng Q, Liu Q, Liu Z, Liu K and Sun H: Catalpol ameliorates hepatic insulin resistance in type 2 diabetes through acting on AMPK/NOX4/PI3K/AKT pathway. Pharmacol Res 130: 466-480, 2018

50. Sun L, Li W, Li W, Xiong L, Li G and Ma R: Astragaloside IV prevents damage to human mesangial cells through the inhibition of the NADPH oxidase/ROS/Akt/NF- $\kappa$ B pathway under high glucose conditions. Int J Mol Med 34: 167-176, 2014.

51. Chen F, Qian LH, Deng B, Liu ZM, Zhao Y and Le YY: Resveratrol protects vascular endothelial cells from high glucose-induced apoptosis through inhibition of NADPH oxidase activation-driven oxidative stress. CNS Neurosci Ther 19: 675-681, 2013.

52. Chowdhury SR, Saleh A, Akude E, Smith DR, Morrow D, Tessler L, Calcutt NA and Fernyhough P: Ciliary neurotrophic factor reverses aberrant mitochondrial bioenergetics through the JAK/STAT pathway in cultured sensory neurons derived from streptozotocin-induced diabetic rodents. Cell Mol Neurobiol 34: 643-649, 2014.

53. Li Q, Lin Y, Wang S, Zhang L and Guo L: GLP-1 inhibits high-glucose-induced oxidative injury of vascular endothelial cells. Sci Rep 7: 8008, 2017.

54. Liu M, Yan L, Liang B, Li Z, Jiang Z, Chu C and Yang J: Hydrogen sulfide attenuates myocardial fibrosis in diabetic rats through the JAK/STAT signaling pathway. Int J Mol Med 41: 1867-1876, 2018.

55. Marrero MB, Banes-Berceli AK, Stern DM and Eaton DC: Role of the JAK/STAT signaling pathway in diabetic nephropathy. Am J Physiol Renal Physiol 290: F762-F768, 2006.

56. Wang X, Shaw S, Amiri F, Eaton DC and Marrero MB: Inhibition of the Jak/STAT signaling pathway prevents the high glucose-induced increase in tgf-beta and fibronectin synthesis in mesangial cells. Diabetes 51: 3505-3509, 2002.

57. Yoshikawa H, Matsubara K, Qian GS, Jackson P, Groopman JD, Manning JE, Harris CC and Herman JG: SOCS-1, a negative regulator of the JAK/STAT pathway, is silenced by methylation in human hepatocellular carcinoma and shows growth-suppression activity. Nat Genet 28: 29-35, 2001.

58. Das A, Salloum FN, Durrant D, Ockaili R and Kukreja RC: Rapamycin protects against myocardial ischemia-reperfusion injury through JAK2-STAT3 signaling pathway. J Mol Cell Cardiol 53: 858-869, 2012.

59. Sun X, Chen RC, Yang ZH, Sun GB, Wang M, Ma XJ, Yang LJ and Sun XB: Taxifolin prevents diabetic cardiomyopathy in vivo and in vitro by inhibition of oxidative stress and cell apoptosis. Food Chem Toxicol 63: 221-232, 2014

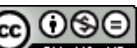

This work is licensed under a Creative Commons Attribution-NonCommercial-NoDerivatives 4.0 International (CC BY-NC-ND 4.0) License. 